\title{
Probing formally forbidden optical transitions in PbSe nanocrystals by time- and energy-resolved transient absorption spectroscopy
}

\author{
Juleon M. Schins, ${ }^{*}$ M. Tuan Trinh, Arjan J. Houtepen, and Laurens D. A. Siebbeles \\ Optoelectronic Materials, Faculty of Applied Sciences, Delft University of Technology, Julianalaan 136, 2628 BL Delft, The Netherlands
}

(Received 1 April 2009; revised manuscript received 2 June 2009; published 27 July 2009)

\begin{abstract}
The first two peaks of the optical extinction spectrum of PbSe nanocrystals in solution have been assigned in the literature to the $1 \mathrm{~S}_{\mathrm{h}} 1 \mathrm{~S}_{\mathrm{e}}$ and $1 \mathrm{P}_{\mathrm{h}} 1 \mathrm{P}_{\mathrm{e}}$ transitions. In the present work we assign the transitions causing extinction in the energy region between these two lowest-energy peaks. Our femtosecond transient absorption data indicate that optical extinction in this region is neither due to Rayleigh scattering nor due to local-field effects but due to the formally forbidden $1 \mathrm{P}_{\mathrm{h}} 1 \mathrm{~S}_{\mathrm{e}}$ and $1 \mathrm{~S}_{\mathrm{h}} 1 \mathrm{P}_{\mathrm{e}}$ transitions.
\end{abstract}

DOI: 10.1103/PhysRevB.80.035323

PACS number(s): 73.21.La, 73.22.Lp

\section{INTRODUCTION}

The nature of the electronic transitions in lead chalcogenides nanocrystals (NCs) is of fundamental importance to their technological application in light-emitting diodes, solidstate lasers, and solar cells. ${ }^{1-4}$ However, the assignment of even the lowest exciton transitions characterizing the $\mathrm{PbSe}$ NC spectrum (see Fig. 1) remains elusive. ${ }^{5-13}$ Apart from the first peak, which has always been attributed to the $1 \mathrm{~S}_{\mathrm{h}} 1 \mathrm{~S}_{\mathrm{e}}$ transition, there is hardly any feature in the spectrum with a straightforward assignment. The assignment of the second peak (located at $0.82 \mathrm{eV}$ for $6.8 \mathrm{~nm}$ diameter $\mathrm{PbSe} \mathrm{NCs}$ ) has been the matter of intense debate during the past years. While the earlier reports assumed the second peak to be of $1 \mathrm{~S}_{\mathrm{h}} 1 \mathrm{P}_{\mathrm{e}}$ character, $6,7,11$ Liljeroth et al. ${ }^{8}$ interpreted their scanning tunneling microscopy data as evidence in favor of the $1 \mathrm{P}_{\mathrm{h}} 1 \mathrm{P}_{\mathrm{e}}$ nature of the second peak, among other reasons because its energy corresponds very closely with the energy difference between their measured energies of the $1 \mathrm{P}_{\mathrm{h}}$ and $1 \mathrm{P}_{\mathrm{e}}$ states.

In a previous publication we have demonstrated conclusively that the second peak cannot be attributed to the $1 \mathrm{~S}_{\mathrm{h}, \mathrm{e}}, 1 \mathrm{P}_{\mathrm{e}, \mathrm{h},}$ transitions. ${ }^{14}$ Using optical pump-probe spectroscopy over a large spectral range we have shown that the presence of $1 \mathrm{~S}$ electrons and holes does not result in a decrease in the transition strength of the second peak but merely to a shift. This suggests that the second peak corresponds to the $1 \mathrm{P}_{\mathrm{h}} 1 \mathrm{P}_{\mathrm{e}}$ transition.

In this contribution we investigate the nature of optical extinction between the first and the second peak, i.e., in the energy region extending from 0.70 to $0.75 \mathrm{eV}$ (see the double question mark in Fig. 1) for PbSe NCs of $6.8 \mathrm{~nm}$ diameter. There is a clear nonzero optical density in this spectral region that cannot be explained by an overlap of the first and second peak; these peaks are too well defined and separated too much. Two Gaussians, accounting for the first two absorption peaks, do not provide an accurate fit of the extinction in this region. ${ }^{5,13}$ It has been suggested before that Rayleigh scattering could be responsible for the nonzero extinction. 5,13 Another possible explanation could be that local-field effects enhance the absorption due to the weak tails of the $1 \mathrm{~S}_{\mathrm{h}} 1 \mathrm{~S}_{\mathrm{e}}$ and $1 \mathrm{P}_{\mathrm{h}} 1 \mathrm{P}_{\mathrm{e}}$ transitions. ${ }^{15,16}$

We show here that Rayleigh scattering and local-field effects cannot explain the optical extinction in the energy region between the $1 \mathrm{~S}_{\mathrm{h}} 1 \mathrm{~S}_{\mathrm{e}}$ and $1 \mathrm{P}_{\mathrm{h}} 1 \mathrm{P}_{\mathrm{e}}$ transitions, most probably it corresponds to the weak-formally forbidden- $-1 \mathrm{~S}_{\mathrm{h}} 1 \mathrm{P}_{\mathrm{e}}$ and $1 \mathrm{P}_{\mathrm{h}} 1 \mathrm{~S}_{\mathrm{e}}$ transitions, termed $1 \mathrm{~S} 1 \mathrm{P}$ transitions in the following.

\section{EXPERIMENT}

Sample preparation and experimental methods are identical to those described earlier. ${ }^{14}$ Oleic acid capped PbSe nanocrystals were synthesized and purified following the recipe of Talapin and Murray. ${ }^{17}$ The NCs were dispersed in tetrachloroethylene for the measurements. The sample is carefully kept free from oxygen and water contamination, both during its preparation and during the measurement. Timeand energy-resolved transient absorption spectroscopy was performed using a commercial Ti:S laser system (MiraLegend USP, Coherent Inc.) and optical parametric amplification for both the pump (Topas-800-fs, Coherent Inc.) and probe beams (Opera, Coherent Inc.). Pump and probe beams overlapped under a small angle $\left(3^{\circ}\right)$ in a cuvette of $10 \mathrm{~mm}$ path length and were imaged onto InGaAs pin photodiodes (Hamamatsu G5853-23, G8605-23). The time resolution is determined (i) by the pulse duration $(<100 \mathrm{fs})$, (ii) by the experimental geometry (pump and probe beams crossing at an angle of $3^{\circ}$ in a cuvette over $10 \mathrm{~mm}$ path length), and (iii)

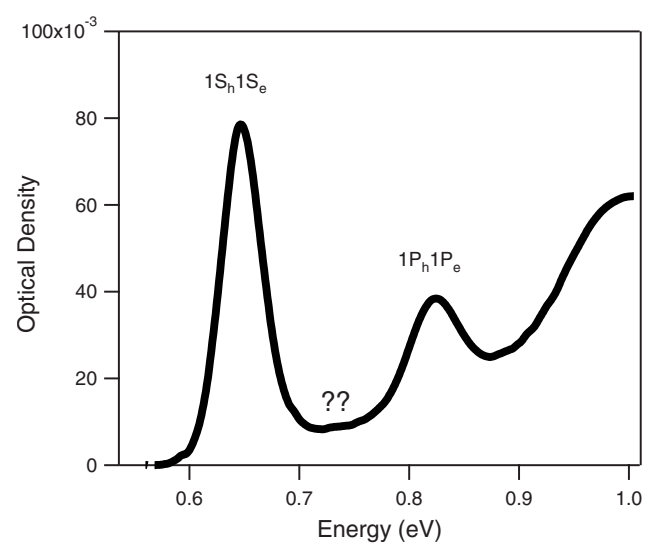

FIG. 1. Optical extinction spectrum of $6.8 \mathrm{~nm}$ PbSe nanocrystals. The current work addresses the energy range $0.70-0.75 \mathrm{eV}$, labeled by the double question mark. 

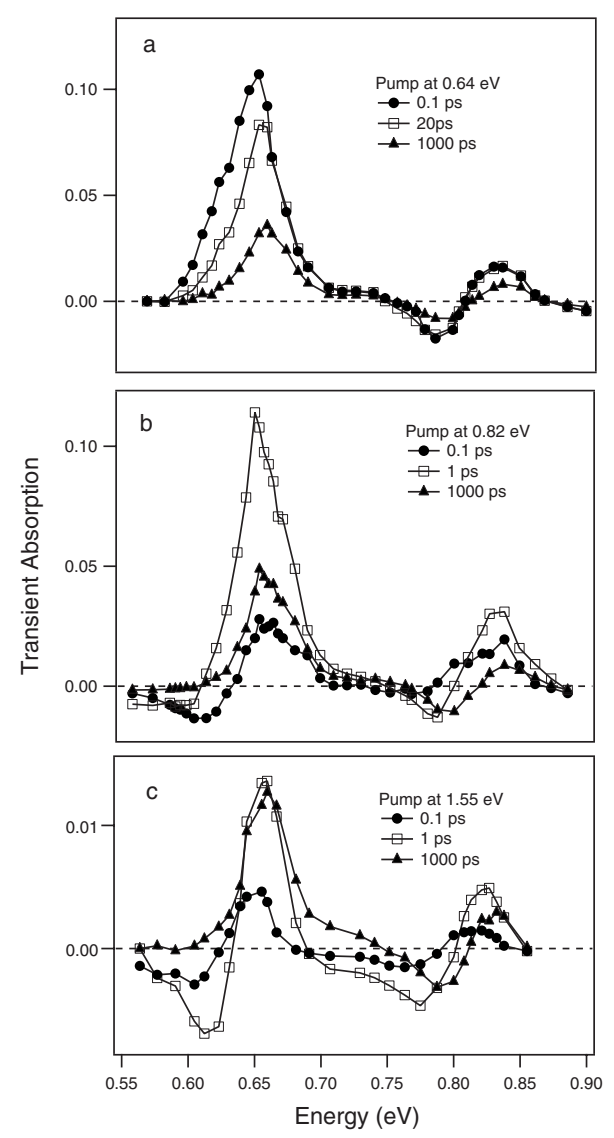

FIG. 2. Transient absorption spectra for PbSe nanocrystals at different delay times and pump-photon energies as indicated. The $1 \mathrm{~S}_{\mathrm{h}} 1 \mathrm{~S}_{\mathrm{e}}$ transition occurs at $0.64 \mathrm{eV}$ and the $1 \mathrm{P}_{\mathrm{h}} 1 \mathrm{P}_{\mathrm{e}}$ transition at $0.82 \mathrm{eV}$.

in case of nondegenerate probing, by dispersion (which induces a difference in cuvette transit times for pump and probe beams). The polarization of the pump and probe was made orthogonal so that pulses of similar photon energy could be separated with polarization filters.

The experimental setup permits variation in the delay time between the pump and probe pulses (denoted by $\tau_{\mathrm{pp}}$ in the following) in a temporal window extending to $1 \mathrm{~ns}$. The measured fractional transmission signals, called transient absorption (TA) in the following, may be written as $T A=\frac{T_{\text {on }}-T_{\text {off }}}{T_{\text {off }}}$, where $T_{\text {on }}$ denotes the probe transmission with pump on and $T_{\text {off }}$ the probe transmission with pump off. With this definition, bleach and emission yield positive TA signals while an increase in absorption yields negative TA signals.

\section{RESULTS}

Figure 2 shows a selection of the measured TA spectra. The probe energy range (horizontal axis) extends from 0.6 through $0.9 \mathrm{eV}$, covering both the $1 \mathrm{~S}_{\mathrm{h}} 1 \mathrm{~S}_{\mathrm{e}}$ and $1 \mathrm{P}_{\mathrm{h}} 1 \mathrm{P}_{\mathrm{e}}$ peaks. The spectra were taken at different pump-probe delay times, as indicated in the figure insets. The pump-photon energy was chosen at $0.64 \mathrm{eV}$ (at the maximum of the $1 \mathrm{~S}_{\mathrm{h}} 1 \mathrm{~S}_{\mathrm{e}}$ peak) in Fig. 2(a), at $0.82 \mathrm{eV}$ (at the maximum of the $1 \mathrm{P}_{\mathrm{h}} 1 \mathrm{P}_{\mathrm{e}}$ peak) in Fig. 2(b), and at $1.55 \mathrm{eV}$ in Fig. 2(c). In order to achieve a high signal-to-noise ratio, the spectra at 0.64 and $0.82 \mathrm{eV}$ pump-photon energy were taken at high fluences: $\sim 10^{-4} \mathrm{~J} \mathrm{~cm}^{-2}$ and $\sim 10^{-3} \mathrm{~J} \mathrm{~cm}^{-2}$, respectively, corresponding to the initial excitation of several $(<10)$ excitons per NC. The pump fluence at $1.55 \mathrm{eV}$ pump-photon energy was $\sim 10^{-6} \mathrm{~J} \mathrm{~cm}^{-2}$, corresponding to an initial production of $\sim 0.1$ excitons per NC.

In a single figure different transient spectra correspond to different pump-probe delay times, as detailed in the figure insets. For the data shown in Fig. 2(c) $(1.55 \mathrm{eV})$ every NC contains at most a single exciton. An initially created hot exciton cools down, typically in 1-5 ps, to the lowest-energy exciton, releasing its excess energy to the crystal lattice. The lowest exciton in $\mathrm{PbSe}$ has a lifetime of about $800 \mathrm{~ns},{ }^{6}$ which is far beyond our range of delay times ( $1 \mathrm{~ns})$. For the data shown in Figs. 2(a) and 2(b) a single NC absorbs on the average more than a single photon, thus leading to the formation of multiple excitons. These multiple excitons decay to the lowest-energy single exciton by Auger recombination on a $50-100$ ps time scale. Hence, at long delay times $\left(\tau_{\mathrm{pp}}>100 \mathrm{ps}\right)$ the high fluence spectra become identical to the low fluence spectra.

The pump fluence of $\sim 10^{-4} \mathrm{~J} \mathrm{~cm}^{-2}$, used in Fig. 2(a), produced on average 3.9 excitons per $\mathrm{NC}$, as was deduced by dividing the initial signal (TA at $\tau_{\mathrm{pp}}=0.5 \mathrm{ps}$ ) over the longtime signal (TA at $\tau_{\mathrm{pp}}=1 \mathrm{~ns}$ ) after spectral integration over the first peak. ${ }^{18}$ For increasing pump-probe delay times the TA signal decreases throughout the measured spectral range. This behavior reflects the Auger decay of the initially $\left(\tau_{\mathrm{pp}}=0.5 \mathrm{ps}\right)$ created multiple excitons to a single exciton $\left(\tau_{\mathrm{pp}}=1 \mathrm{~ns}\right)$. The $1 \mathrm{~S}_{\mathrm{h}} 1 \mathrm{~S}_{\mathrm{e}}$ exciton population produced by the pump causes a bleach at the $1 \mathrm{~S}_{\mathrm{h}} 1 \mathrm{~S}_{\mathrm{e}}$ transition, with a peak, that is, slightly blueshifted with respect to the ground-state absorption. A blueshift in the TA spectrum implies a redshift in the excited-state absorption. Figure 2(a) clearly shows no bleach at the $1 \mathrm{P}_{\mathrm{h}} 1 \mathrm{P}_{\mathrm{e}}$ transition but only a redshift, giving rise to the typical antisymmetric TA profile. ${ }^{14}$ Interestingly, the TA signal is positive in the region $0.70-0.75 \mathrm{eV}$. As will be discussed below, this excludes Rayleigh scattering as a possible mechanism for light extinction.

Figure 2(b) shows the TA spectra for a pump-photon energy of $0.82 \mathrm{eV}$, which is at the maximum of the $1 \mathrm{P}_{\mathrm{h}} 1 \mathrm{P}_{\mathrm{e}}$ absorption peak. At the shortest pump-probe delay times the $1 \mathrm{~S}_{\mathrm{h}} 1 \mathrm{~S}_{\mathrm{e}}$ absorption feature is shifted to the red, exhibiting a bleach that increases with time and a shift that decreases with time. The opposite holds true for the $1 \mathrm{P}_{\mathrm{h}} 1 \mathrm{P}_{\mathrm{e}}$ feature, as time progresses, the bleach decreases. At $\tau_{\mathrm{pp}}=1 \mathrm{~ns}$ the bleach has disappeared completely. The antisymmetric wiggle seen in the spectrum [Fig. 2(b), in the region $0.75-0.87 \mathrm{eV}$ ] is the signature of a mere spectral shift of an absorption feature. These features are readily understood in terms of a decay of the initially excited $1 \mathrm{P}_{\mathrm{h}} 1 \mathrm{P}_{\mathrm{e}}$ population to the $1 \mathrm{~S}_{\mathrm{h}} 1 \mathrm{~S}_{\mathrm{e}}$ state. The decay time is obtained from the decrease in the integrated $1 \mathrm{P}_{\mathrm{h}} 1 \mathrm{P}_{\mathrm{e}}$ bleach or from the increase in the integrated $1 \mathrm{~S}_{\mathrm{h}} 1 \mathrm{~S}_{\mathrm{e}}$ bleach. Both integrations show an exponential decay characterized by a cooling time of $1.2 \mathrm{ps}$. This is close to decay times reported previously, ${ }^{19,20}(\sim 1.5 \mathrm{ps})$ where no spectral integration was used.

Figure 2(c) shows the transient spectra for a pump-photon energy of $1.55 \mathrm{eV}$. The most distinct feature is the initial 


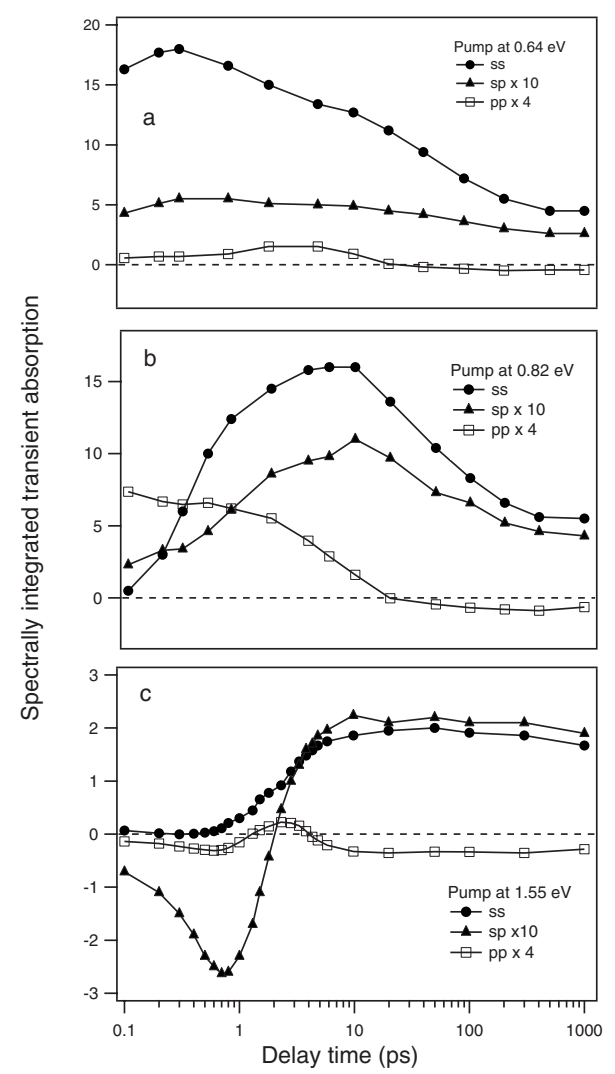

FIG. 3. Spectrally integrated TA signals of PbSe NCs, showing the populations of the $1 \mathrm{~S}_{\mathrm{h}} 1 \mathrm{~S}_{\mathrm{e}}$ (marked ss), intermediate (sp), and $1 \mathrm{P}_{\mathrm{h}} 1 \mathrm{P}_{\mathrm{e}}$ (pp) levels for three different pump-photon energies: 0.64 $\mathrm{eV}\left(1 \mathrm{~S}_{\mathrm{h}} 1 \mathrm{~S}_{\mathrm{e}}\right), 0.82 \mathrm{eV}\left(1 \mathrm{P}_{\mathrm{h}} 1 \mathrm{P}_{\mathrm{e}}\right)$, and $1.55 \mathrm{eV}$. The intermediate signals have been multiplied by 10 , the $1 \mathrm{P}_{\mathrm{h}} 1 \mathrm{P}_{\mathrm{e}}$ signals by 4 . These multiplication factors correspond to the relative strengths of the absorptive transitions as shown in Fig. 1; hence the curves represent normalized relative TA signals. Note that the vertical scales are not identical in the three figures.

"pure shift" (absence of bleach) of both the $1 \mathrm{~S}_{\mathrm{h}} 1 \mathrm{~S}_{\mathrm{e}}$ and $1 \mathrm{P}_{\mathrm{h}} 1 \mathrm{P}_{\mathrm{e}}$ transitions. This is due to the fact that the initially created hot exciton leaves the population of both the $1 \mathrm{~S}_{\mathrm{h}} 1 \mathrm{~S}_{\mathrm{e}}$ and $1 \mathrm{P}_{\mathrm{h}} 1 \mathrm{P}_{\mathrm{e}}$ transitions unchanged. Hence no bleach occurs but only a shift of the energy levels as a consequence of the spectator exciton. As the hot excitons decay to the $1 \mathrm{~S}_{\mathrm{h}} 1 \mathrm{~S}_{\mathrm{e}}$ state, the initial $1 \mathrm{~S}_{\mathrm{h}} 1 \mathrm{~S}_{\mathrm{e}}$ shift is gradually converted into a $1 \mathrm{~S}_{\mathrm{h}} 1 \mathrm{~S}_{\mathrm{e}}$ bleach; since at $\tau_{\mathrm{pp}}=1 \mathrm{~ns}$ there is no $1 \mathrm{P}_{\mathrm{h}} 1 \mathrm{P}_{\mathrm{e}}$ population, the $1 \mathrm{P}_{\mathrm{h}} 1 \mathrm{P}_{\mathrm{e}}$ feature shows a pure shift just as at short pump-probe delay times.

More insight can be obtained from the data by spectral integration over specific energy domains, corresponding to the $1 \mathrm{~S}_{\mathrm{h}} 1 \mathrm{~S}_{\mathrm{e}}$ feature $(0.56-0.70 \mathrm{eV})$, the intermediate domain $(0.70-0.75 \mathrm{eV})$, and the $1 \mathrm{P}_{\mathrm{h}} 1 \mathrm{P}_{\mathrm{e}}$ feature $(0.75-0.87 \mathrm{eV})$. The results are shown as a function of delay time in Fig. 3. For the sake of clarity, the $1 \mathrm{P}_{\mathrm{h}} 1 \mathrm{P}_{\mathrm{e}}$ transients have been multiplied by a factor 4 and the intermediate transients with a factor 10 compared to the $1 \mathrm{~S}_{\mathrm{h}} 1 \mathrm{~S}_{\mathrm{e}}$ transients, in order to account for the differences in optical density between these transitions. In discussing these traces one should keep in mind that they depend to some extent on the exact bounds chosen for the integration. The error margin on the integrated data shown in Fig. 3 is less than 5\%. This error is not due to experimental noise but due to the uncertainty of the integration bounds and these on their turn are not uniquely defined as the energies of the involved transitions are subject to excitonic shifts.

Figure 3(a) displays the temporal behavior of the three features in the case of a pump-photon energy of $0.64 \mathrm{eV}$. The $1 \mathrm{~S}_{\mathrm{h}} 1 \mathrm{~S}_{\mathrm{e}}$ feature shows the Auger decay of the multiply excited $1 \mathrm{~S}_{\mathrm{h}} 1 \mathrm{~S}_{\mathrm{e}}$ excitons; initially on the average 3.9 excitons per NC are excited; the biexciton life time is on the order of 100 ps. $^{18,21}$ The initial bleach of the intermediate feature points to the involvement of a $1 \mathrm{~S}$ state. There is no appreciable $1 \mathrm{P}_{\mathrm{h}} 1 \mathrm{P}_{\mathrm{e}}$ bleach, which is consistent with the absence of $1 \mathrm{~S}$ character in the $1 \mathrm{P}_{\mathrm{h}} 1 \mathrm{P}_{\mathrm{e}}$ states.

Figure 3(b) displays the temporal behavior of the three features in the case of a pump-photon energy of $0.82 \mathrm{eV}$. The $1 \mathrm{P}_{\mathrm{h}} 1 \mathrm{P}_{\mathrm{e}}$ exciton is populated directly; it decays monotonously due to population transfer to the $1 \mathrm{~S}$ electron and hole states. The rise time of the $1 \mathrm{~S}_{\mathrm{h}} 1 \mathrm{~S}_{\mathrm{e}}$ bleach $(\sim 1 \mathrm{ps})$ reflects the time needed for the initially excited $1 \mathrm{P}_{\mathrm{h}} 1 \mathrm{P}_{\mathrm{e}}$ population to decay to the $1 \mathrm{~S}_{\mathrm{h}} 1 \mathrm{~S}_{\mathrm{e}}$ state. The subsequent decay of the signal is due to Auger recombination of multiple excitons. The intermediate feature starts out with a "immediate" (resolution-limited) bleach, increasing until 10 ps. The immediate bleach implies involvement of a 1P state in the intermediate transition; the subsequent rise in the signal is due to decay of $1 \mathrm{P}$ states to $1 \mathrm{~S}$ states, the latter contributing a factor 3 more to the bleach (since the $1 \mathrm{~S}$ manifold is eightfold degenerated, versus the 24-fold degeneracy of the 1P manifold). The decrease after $10 \mathrm{ps}$ is due to Auger recombination of the $1 \mathrm{~S}$ states, which have been shown above [Fig. 3(a)] to be involved in the intermediate transitions, too.

Figure 3(c) displays the temporal behavior of the three features in the case of a pump-photon energy of $1.55 \mathrm{eV}$. The $1 \mathrm{~S}_{\mathrm{h}} 1 \mathrm{~S}_{\mathrm{e}}$ bleach rises slower than in the previous cases $(\sim 2.5 \mathrm{ps})$ because the initial population has higher energy and therefore needs more time to decay to the $1 \mathrm{~S}_{\mathrm{h}} 1 \mathrm{~S}_{\mathrm{e}}$ state. The integrated $1 \mathrm{P}_{\mathrm{h}} 1 \mathrm{P}_{\mathrm{e}}$ signal is very low at all times; it decreases initially, peaks at $2 \mathrm{ps}$, and decreases again at longer times. The small bleach at 2 ps can be understood as a transient population of the $1 \mathrm{P}_{\mathrm{h}} 1 \mathrm{P}_{\mathrm{e}}$ state. This transient population can be expected when an initially excited hot exciton cools down to the $1 \mathrm{~S}_{\mathrm{h}} 1 \mathrm{~S}_{\mathrm{e}}$ state. Most importantly, the intermediate feature now behaves quite differently from the $1 \mathrm{~S}_{\mathrm{h}} 1 \mathrm{~S}_{\mathrm{e}}$ feature, showing an initial increase in absorption followed by a bleach at the time that most of the excited population has decayed to the lowest exciton state $\left(\tau_{\mathrm{pp}} \sim 10 \mathrm{ps}\right)$. The fact that the intermediate range TA signal in Fig. 3(c) has an extreme at $\tau_{\mathrm{pp}} \sim 1 \mathrm{ps}$ is not due to population dynamics. At variance with the situation in Figs. 2(a) and 2(b), where the pump and probe energies are nearly degenerate, in the case of $1.55 \mathrm{eV}$ pump photons the dispersion in the sample (10 $\mathrm{mm}$ pathway) largely determines the temporal experimental resolution. Hence, the extreme at $\tau_{\mathrm{pp}} \sim 1 \mathrm{ps}$ is due to the temporal resolution and the increase in oscillator strength of the intermediate energy states must be considered as instantaneous with the $1.55 \mathrm{eV}$ pump pulse. Due to the energy differences between pump and probe there is quite some dispersion in the sample $(10 \mathrm{~mm})$. This results in there not being a single, uniquely defined "experimental reso- 
lution." Every pump-probe combination has its own experimental resolution, which is optimal at degeneracy (equal pump and probe photon energies), and worsens for increasing energy difference between the pump and probe photon. This has to be kept in mind when evaluating the temporal traces.

\section{DISCUSSION}

As mentioned in Sec. I, there are several different ways to explain the extinction feature in the energy range 0.70-0.75 eV (Fig. 1): (i) Rayleigh scattering, (ii) local-field effects, (iii) satellites of the allowed $1 \mathrm{~S}_{\mathrm{h}} 1 \mathrm{~S}_{\mathrm{e}}$ and $1 \mathrm{P}_{\mathrm{h}} 1 \mathrm{P}_{\mathrm{e}}$ transitions, and (iv) the formally forbidden optical $1 \mathrm{~S}_{\mathrm{h}} 1 \mathrm{P}_{\mathrm{e}}$ and $1 \mathrm{P}_{\mathrm{h}} 1 \mathrm{~S}_{\mathrm{e}}$ transitions. Rayleigh scattering ${ }^{5}$ and local-field effects ${ }^{15}$ have been suggested in the literature in connection with the PbSe NC absorption spectra but these effects start to play a significant role at energies well beyond the $1 \mathrm{P}_{\mathrm{h}} 1 \mathrm{P}_{\mathrm{e}}$ transition, with a takeoff at approximately $1.4 \mathrm{eV}$ for $6.8 \mathrm{~nm}$ NCs. ${ }^{5}$ Moreover, Rayleigh scattering can be excluded on the basis of the sign of the observed TA signal; the TA signal in the region $0.70-0.75 \mathrm{eV}$ [see Fig. 2(a)] is positive while Rayleigh scattering would imply a negative TA signal. When the NCs are excited their polarizability increases and so does the difference in polarizability with respect to the surrounding capping and solution molecules (oleic acid and tetrachloroethylene, respectively). An increased difference in polarizability leads to an increase in Rayleigh scattering, a decrease in the transmitted probe light, and consequently a negative TA signal. Finally, for our size nanocrystals the Rayleigh cross section is about four orders of magnitude smaller than the absorption cross section at the $1 \mathrm{~S}_{\mathrm{h}} 1 \mathrm{~S}_{\mathrm{e}}$ transition. ${ }^{22}$ Hence, our positive TA signal in the region $0.70-0.75 \mathrm{eV}$ excludes Rayleigh scattering as the mechanism responsible for light extinction.

Assignment of the intermediate extinction in terms of satellites of the $1 \mathrm{~S}_{\mathrm{h}} 1 \mathrm{~S}_{\mathrm{e}}$ states is not likely either. In order to explain our data in terms of $1 \mathrm{~S}_{\mathrm{h}} 1 \mathrm{~S}_{\mathrm{e}}$ satellites one must postulate the existence of an alternative $1 \mathrm{~S}$ state, $0.1 \mathrm{eV}$ (roughly half the value of the separation between the $1 \mathrm{~S}_{\mathrm{h}} 1 \mathrm{~S}_{\mathrm{e}}$ and $1 \mathrm{P}_{\mathrm{h}} 1 \mathrm{P}_{\mathrm{e}}$ transitions) higher in energy than the main $1 \mathrm{~S}$ state. This energy of $0.1 \mathrm{eV}$ may not be divided equally among the two $1 \mathrm{~S}$ states supposedly configuring the intermediate transition because the intermediate transition bleaches instantaneously when pumped at the $1 \mathrm{~S}_{\mathrm{h}} 1 \mathrm{~S}_{\mathrm{e}}$ transition [Fig. 3(a)]. Theoretical calculations-verified experimentally ${ }^{23}$ - have shown that the intervalley splitting in $6.12 \mathrm{~nm}$ diameter PbSe NCs has a value of only $18 \mathrm{meV} .^{24}$ Hence the intervalley splitting cannot account for the existence of $1 \mathrm{~S}$ satellites $0.1 \mathrm{eV}$ higher in energy than the $1 \mathrm{~S}$ states which give rise to the $1 \mathrm{~S}_{\mathrm{h}} 1 \mathrm{~S}_{\mathrm{e}}$ absorption peak.

Extinction in the intermediate energy region (0.70-0.75 eV, Fig. 1) is more plausibly explained by absorption of the formally forbidden 1S1P transitions. As shown in Fig. 3, the intermediate energy region is bleached instantaneously when pumped at $0.64 \mathrm{eV}\left[1 \mathrm{~S}_{\mathrm{h}} 1 \mathrm{~S}_{\mathrm{e}}\right.$ transition, Fig. 3(a)] or at $0.82 \mathrm{eV}\left[1 \mathrm{P}_{\mathrm{h}} 1 \mathrm{P}_{\mathrm{e}}\right.$ transitions, Fig. 3(b)]. This implies that the optical transitions in the intermediate energy region share electronic levels with both the $1 \mathrm{~S}_{\mathrm{h}} 1 \mathrm{~S}_{\mathrm{e}}$ and
$1 \mathrm{P}_{\mathrm{h}} 1 \mathrm{P}_{\mathrm{e}}$ transition. Hence, they correspond to the $1 \mathrm{~S}_{\mathrm{h}} 1 \mathrm{P}_{\mathrm{e}}$ and $1 \mathrm{P}_{\mathrm{h}} 1 \mathrm{~S}_{\mathrm{e}}$ transitions.

Theory shows that the $1 \mathrm{~S} 1 \mathrm{P}$ transitions are forbidden in perfectly spherical nanocrystals in the absence of both surface charge and a ground-state dipole moment. ${ }^{9,12,25-29}$ The nanocrystals investigated here might differ from this ideal in several respects, including deviations from perfect symmetry, surface passivation chemistry, nonstoichiometric composition in terms of the constituent $\mathrm{Pb}$ and $\mathrm{Se}$ atoms, ${ }^{30}$ surface charges, ${ }^{13}$ defects, and vacancies. E.g., it has been shown that $\mathrm{PbSe}$ NCs possess a ground-state dipole moment in excess of 300 Debye. $^{31}$

Interestingly, the oscillator strength of the weakly allowed $1 \mathrm{P}_{\mathrm{h}} 1 \mathrm{~S}_{\mathrm{e}}$ and $1 \mathrm{~S}_{\mathrm{h}} 1 \mathrm{P}_{\mathrm{e}}$ transitions are strongly enhanced by excitation with $1.55 \mathrm{eV}$ pump photons. In Figs. 2(c) and 3(c) the TA signal at the intermediate energy region becomes negative at short times. The negative signal reflects the increase in oscillator strength of the $1 \mathrm{P}_{\mathrm{h}} 1 \mathrm{~S}_{\mathrm{e}}$ and $1 \mathrm{~S}_{\mathrm{h}} 1 \mathrm{P}_{\mathrm{e}}$ transitions following absorption of a $1.55 \mathrm{eV}$ pump photon. This can be attributed to ulterior relaxation of the forbidden nature of the ideal SP transition.

A simple simulation might illustrate one aspect of the behavior of the normalized traces shown in Fig. 3(b), i.e., this model is not meant to account for the details. In order to keep the model as simple as possible we assume the $1 \mathrm{P} \rightarrow 1 \mathrm{~S}$ decay rates of electrons and holes to be identical; however, this is not imposed by the experimental data. The populations of the $1 \mathrm{~S}_{\mathrm{h}} 1 \mathrm{~S}_{\mathrm{e}}$, the $1 \mathrm{~S} 1 \mathrm{P}$, and the $1 \mathrm{P}_{\mathrm{h}} 1 \mathrm{P}_{\mathrm{e}}$ exciton states, denoted by $n_{\mathrm{ss}}, n_{\mathrm{sp}}$, and $n_{\mathrm{pp}}$, respectively, decay as follows: ${ }^{32}$

$$
\begin{gathered}
\dot{n}_{\mathrm{pp}}=-2 \gamma n_{\mathrm{pp}}, \\
\dot{n}_{\mathrm{sp}}=2 \gamma n_{\mathrm{pp}}-\gamma n_{\mathrm{sp}}, \\
\dot{n}_{\mathrm{ss}}=\gamma n_{\mathrm{sp}}-\xi\left(n_{\mathrm{ss}}-1\right) n_{\mathrm{ss}}^{2} .
\end{gathered}
$$

Here $\gamma$ is the rate at which a $1 \mathrm{P}$ state decays to a $1 \mathrm{~S}$ state and $\xi$ is the rate for Auger recombination of multiple $1 \mathrm{~S}_{\mathrm{h}} 1 \mathrm{~S}_{\mathrm{e}}$ excitons; for the present argument it is not relevant whether Auger recombination occurs with the second or third power of the exciton density (the above expression displays a third power dependence). ${ }^{32}$ Independently of the occurrence of cross transitions,${ }^{14}$ the $1 \mathrm{~S} 1 \mathrm{P}$ bleach produced in the by these populations can be written as follows:

$$
\left(\frac{\Delta \alpha}{\alpha}\right)_{\mathrm{sp}}=\frac{n_{\mathrm{pp}}}{24}+\frac{n_{\mathrm{sp}}}{12}+\frac{n_{\mathrm{ss}}}{8},
$$

where the factors 8 and 24 reflect the degeneracies of the $\mathrm{S}$ and $\mathrm{P}$ states, respectively. Using $\gamma=0.2 \mathrm{ps}^{-1}$ and $\xi=0.003 \mathrm{ps}^{-1}$, and the initial condition that each $\mathrm{NC}$ contains $41 \mathrm{P}_{\mathrm{h}} 1 \mathrm{P}_{\mathrm{e}}$ excitons immediately after excitation, ${ }^{14}$ this leads to the solution shown in Fig. 4. The initial $1 \mathrm{P}_{\mathrm{h}} 1 \mathrm{P}_{\mathrm{e}}$ exciton population of 4 can only be reached experimentally upon using high fluence.

It reproduces the essential features seen in the experimental data [see Fig. 3(b)], although not the details; this can be expected given the simplifications inherent in the model. 


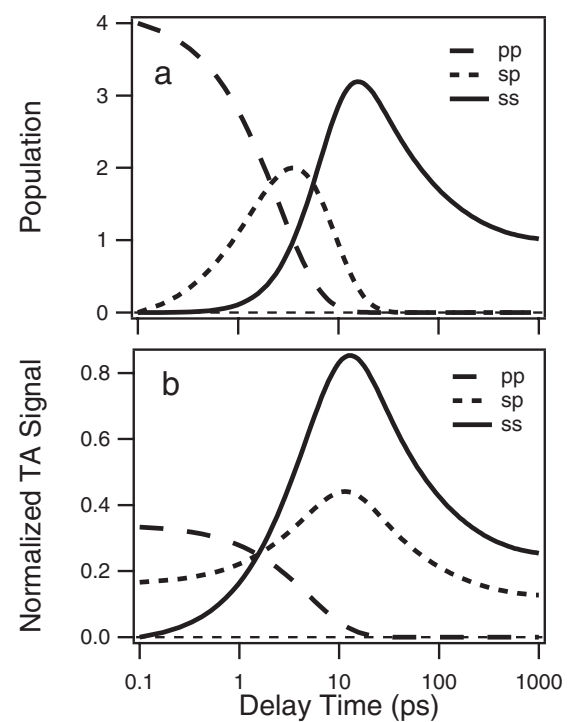

FIG. 4. (a) Population and (b) normalized TA signal as a function of time, as expected for the simplified model system described in the text.

Summarizing, we have shown that extinction in the absorption spectrum of PbSe nanocrystals in the intermediate energy range $(0.70-0.75 \mathrm{eV}$, see the double question mark in Fig. 1) is neither due to Rayleigh scattering, nor due to localfield effects but due to the $1 \mathrm{~S}_{\mathrm{h}} 1 \mathrm{P}_{\mathrm{e}}$ and $1 \mathrm{P}_{\mathrm{h}} 1 \mathrm{~S}_{\mathrm{e}}$ transitions. These transitions are optically forbidden in ideally symmetric, uncharged nanocrystals, lacking a ground-state dipole moment.

In order to uncover whether the intermediate extinction feature in the ground-state absorption spectrum is due to either $1 \mathrm{~S}_{\mathrm{h}} 1 \mathrm{~S}_{\mathrm{e}}$ or $1 \mathrm{P}_{\mathrm{h}} 1 \mathrm{P}_{\mathrm{e}}$ transitions, three experiments were performed. In the first two experiments the sample was pumped at exactly those transitions. In order to obtain a high signal-to-noise ratio we chose to pump at large fluence, using the fact that the pump photons create specific excitons (i.e., $1 \mathrm{~S}_{\mathrm{h}} 1 \mathrm{~S}_{\mathrm{e}}$ and $1 \mathrm{P}_{\mathrm{h}} 1 \mathrm{P}_{\mathrm{e}}$ excitons, respectively). In a third experiment the sample was pumped at a photon energy much higher than the $1 \mathrm{~S}_{\mathrm{h}} 1 \mathrm{~S}_{\mathrm{e}}$ and $1 \mathrm{P}_{\mathrm{h}} 1 \mathrm{P}_{\mathrm{e}}$ transitions. In this case one does not know what exciton is created. We chose to pump at low fluence, in order to verify what effect a single, yet unknown, hot exciton state has on the energies of the lower transitions. As has been shown previously by Trinh $e t$ $a l .{ }^{18}$ spectral integration is imperative in order to estimate excitonic populations in the presence of excitonic shifts. These exciton-dependent energy shifts are due to multiexcitonic Coulomb effects.

A positive transient absorption signal in the intermediate energy regime $(0.7-0.75 \mathrm{eV})$, although small, clearly exceeds the noise in the data. Yet even if the signal were zero, our main conclusion (concerning the electronic nature of the intermediate energy extinction feature) would still hold; if the extinction feature were due to Rayleigh scattering, then the signal should have been negative. The signal is not negative, as clearly shown in Fig. 2(a). Ergo, the extinction in the ground-state absorption spectrum cannot be explained by Rayleigh scattering. Since our experiments exclude Rayleigh scattering as an origin for extinction in the intermediate energy regime of the ground-state absorption spectrum, the lower energy part of the absorption spectrum can be fully accounted for by the four transitions $1 \mathrm{~S}_{\mathrm{h}} 1 \mathrm{~S}_{\mathrm{e}}, 1 \mathrm{P}_{\mathrm{h}} 1 \mathrm{~S}_{\mathrm{e}}$, $1 \mathrm{~S}_{\mathrm{h}} 1 \mathrm{P}_{\mathrm{e}}$, and $1 \mathrm{P}_{\mathrm{h}} 1 \mathrm{P}_{\mathrm{e}}$, and no background contribution needs to be invoked. At higher energies the cross section of Rayleigh scattering increases significantly. However, a full description of the ground-state extinction spectrum at higher energies is beyond the scope of our work.

Finally, we report that the oscillator strengths of the 1S1P transitions are significantly enhanced following excitation with $1.55 \mathrm{eV}$ photons.

\section{ACKNOWLEDGMENTS}

This work is part of the Joint Solar Programme (JSP) of the Stichting voor Fundamenteel Onderzoek der Materie (FOM). JSP is cofinanced by "Gebied Chemische Wetenschappen" of the Dutch Organisation for Scientific Research (NWO) and by Stichting Shell Research. We furthermore acknowledge the 3TU Centre for Sustainable Energy Technologies (Federation of the three Universities of Technology) and the Division of Chemical Sciences of NWO for the VICI Award No. 700.53.443. We thank A. Franceschetti and F.C. Grozema for helpful discussions.

*j.m.schins@tudelft.nl

${ }^{1}$ P. V. Kamat, J. Phys. Chem. C 112, 18737 (2008).

${ }^{2}$ S. Coe, W. K. Woo, M. Bawendi, and V. Bulovic, Nature (London) 420, 800 (2002).

${ }^{3}$ V. I. Klimov, A. A. Mikhailovsky, S. Xu, A. Malko, J. A. Hollingsworth, C. A. Leatherdale, H. J. Eisler, and M. G. Bawendi, Science 290, 314 (2000).

${ }^{4}$ A. J. Nozik, Annu. Rev. Phys. Chem. 52, 193 (2001).

${ }^{5}$ R. Koole, G. Allan, C. Delerue, A. Meijerink, D. Vanmaekelbergh, and A. J. Houtepen, Small 4, 127 (2008).

${ }^{6}$ B. L. Wehrenberg, C. J. Wang, and P. Guyot-Sionnest, J. Phys. Chem. B 106, 10634 (2002).

${ }^{7}$ B. L. Wehrenberg and P. Guyot-Sionnest, J. Am. Chem. Soc.

125, 7806 (2003).

${ }^{8}$ P. Liljeroth, P. A. Z. van Emmichoven, S. G. Hickey, H. Weller, B. Grandidier, G. Allan, and D. Vanmaekelbergh, Phys. Rev. Lett. 95, 086801 (2005).

${ }^{9}$ J. M. An, A. Franceschetti, S. V. Dudiy, and A. Zunger, Nano Lett. 6, 2728 (2006).

${ }^{10}$ J. J. Peterson, L. Huang, C. Delerue, G. Allan, and T. D. Krauss, Nano Lett. 7, 3827 (2007).

${ }^{11}$ J. M. Harbold, H. Du, T. D. Krauss, K. S. Cho, C. B. Murray, and F. W. Wise, Phys. Rev. B 72, 195312 (2005).

${ }^{12}$ H. Du, C. L. Chen, R. Krishnan, T. D. Krauss, J. M. Harbold, F. W. Wise, M. G. Thomas, and J. Silcox, Nano Lett. 2, 1321 (2002). 
${ }^{13}$ J. M. An, A. Franceschetti, and A. Zunger, Phys. Rev. B 76, 161310 (2007).

${ }^{14}$ M. T. Trinh, A. J. Houtepen, J. M. Schins, J. Piris, and L. D. A. Siebbeles, Nano Lett. 8, 2112 (2008).

${ }^{15}$ I. Moreels and Z. Hens, Small 4, 1866 (2008).

${ }^{16}$ R. Koole, G. Allan, C. Delerue, D. Vanmaekelbergh, A. J. Houtepen, and A. Meijerink, Small 4, 1869 (2008).

${ }^{17}$ D. V. Talapin and C. B. Murray, Science 310, 86 (2005).

${ }^{18}$ M. T. Trinh, A. J. Houtepen, J. M. Schins, T. Hanrath, J. Piris, W. Knulst, A. P. L. M. Goossens, and L. D. A. Siebbeles, Nano Lett. 8, 1713 (2008).

${ }^{19}$ R. D. Schaller, J. M. Pietryga, S. V. Goupalov, M. A. Petruska, S. A. Ivanov, and V. I. Klimov, Phys. Rev. Lett. 95, 196401 (2005).

${ }^{20}$ C. Bonati, A. Cannizzo, D. Tonti, A. Tortschanoff, F. van Mourik, and M. Chergui, Phys. Rev. B 76, 033304 (2007).

${ }^{21}$ V. I. Klimov, J. A. McGuire, R. D. Schaller, and V. I. Rupasov, Phys. Rev. B 77, 195324 (2008).

${ }^{22}$ I. Moreels, Doctoral thesis, University of Gent, 2009.
${ }^{23}$ J. M. Harbold and F. W. Wise, Phys. Rev. B 76, 125304 (2007).

${ }^{24}$ J. M. An, A. Franceschetti, and A. Zunger, Nano Lett. 7, 2129 (2007).

${ }^{25}$ S. V. Gaponenko, Optical Properties of Semiconductor Nanocrystals (Cambridge University Press, Cambridge, 1998), p. 245.

${ }^{26}$ D. J. Norris, in Semiconductor and Metal Nanocrystals, edited by V. I. Klimov (Marcel Dekker, New York, 2004), pp. 65-102.

${ }^{27}$ C. Delerue and M. Lannoo, Nanostructures: Theory and Modelling (Springer-Verlag, Berlin, 2004).

${ }^{28}$ G. Allan and C. Delerue, Phys. Rev. B 70, 245321 (2004).

${ }^{29}$ I. Kang and F. W. Wise, J. Opt. Soc. Am. B 14, 1632 (1997).

${ }^{30}$ I. Moreels, B. Fritzinger, J. C. Martins, and Z. Hens, J. Am. Chem. Soc. 130, 15081 (2008).

${ }^{31}$ M. Klokkenburg, A. J. Houtepen, R. Koole, J. W. J. de Folter, B. H. Erne, E. van Faassen, and D. Vanmaekelbergh, Nano Lett. 7, 2931 (2007).

${ }^{32}$ A. V. Barzykin and M. Tachiya, J. Phys.: Condens. Matter 19, 065105 (2007). 